\title{
Coordinateurs, contributeurs, mécènes et remerciements
}

\section{Les coordinateurs}

Cet ouvrage collectif a été écrit par 19 personnes dont les noms figurent en tête des chapitres et ci-dessous. La coordination en a été assurée par Robin Kaiser, Michèle Leduc et Hélène Perrin.

\section{Robin Kaiser}

Robin Kaiser est directeur de recherche au CNRS. Il a débuté sa carrière en physique atomique à l'École normale supérieure par une thèse dirigée par Alain Aspect, dans le groupe de Claude Cohen-Tannoudji. Il a ensuite effectué un séjour postdoctoral à l'université de Harvard dans le groupe de Gerald Gabrielse, avant de rejoindre, sur un poste de chargé de recherche au CNRS, Alain Aspect pour démarrer une nouvelle activité en atomes froids à l'Institut d'Optique. Depuis 1996, Robin Kaiser dirige l'équipe «Atomes froids » à l'Institut de physique de Nice. Ses travaux de recherche concernent essentiellement la diffusion de lumière, combinant la

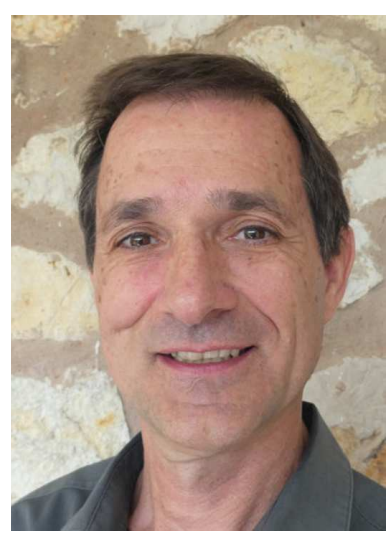
physique des atomes froids avec celle de la physique mésoscopique, de la localisation de lumière et de l'optique quantique. Il a initié des études de corrélations d'intensité en astrophysique, reprenant ainsi les études historiques de Hanbury 
Brown et Twiss avec les outils modernes de l'optique quantique. Il est aussi le directeur du GDR Atomes froids depuis sa création.

\section{Michèle Leduc}

Michèle Leduc est directrice de recherche émérite au CNRS. Sa carrière en physique atomique s'est essentiellement déroulée à l'École normale supérieure à Paris, dans le Laboratoire LKB qui porte le nom de ses fondateurs Alfred Kastler (lauréat du prix Nobel en 1966) et Jean Brossel. En 1993 elle rejoint l'équipe qui travaille sur le refroidissement laser dirigée par Claude CohenTannoudji, lauréat du prix Nobel en 1997. Ses travaux de recherche les plus récents portent sur les condensats de Bose-Einstein d'hélium métastable. Elle coordonne aujourd'hui les activités pour le rayonnement extérieur de SIRTEQ, le réseau de recherche sur les technologies quantiques de la région Île-de-France. Elle est aussi éditrice de collections d'ouvrages de science pour le CNRS et pour EDP-Sciences. Elle assure des missions de réflexion sur l'éthique dans diverses instances telles que le COMETS (Comité d'éthique du CNRS).

\section{Hélène Perrin}

Hélène Perrin est directrice de recherche au CNRS. Après une thèse au Laboratoire Kastler-Brossel sous la direction de Christophe Salomon sur le refroidissement laser d'atomes dans un piège optique et un séjour postdoctoral au CEA sur les gaz d'électrons bidimensionnels avec Christian Glattli, elle a été recrutée au CNRS au sein du Laboratoire de physique des lasers de l'Université Paris-Nord, où elle dirige l'équipe "Condensats de Bose-Einstein ». Sa recherche porte sur les condensats de Bose-Einstein confinés dans des pièges radiofréquences et plus particulièrement sur leurs propriétés superfluides. Elle enseigne à l'École normale

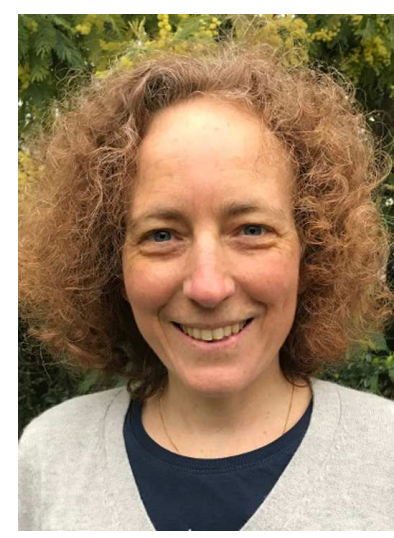
supérieure et à l'Université de Paris, et est régulièrement invitée à donner des cours dans des écoles d'été internationales comme l'école des Houches. Elle coordonne avec Pascal Simon l'axe "Simulation quantique» de SIRTEQ et est membre du bureau du GDR « Atomes froids». 


\section{Les contributeurs}

Les personnes suivantes ont contribué à la rédaction de cet ouvrage : Baptiste Allard, Juliette Billy, Nadia Bouloufa-Maafa, Nicolas Cherroret, Daniel Comparat, Olivier Dulieu, Laurent Hilico, Vincent Josse, Robin Kaiser, Martina Knoop, Bruno Laburthe, Thierry Lahaye, Michèle Leduc, Hans Lignier, Jérôme Lodewyck, Franck Pereira dos Santos, Hélène Perrin, Goulven Quéméner, Jakob Reichel. Ces contributeurs poursuivent leurs recherches dans des laboratoires associés au CNRS et pour la plupart à diverses institutions universitaires. Les coordinateurs remercient vivement tous ces auteurs pour leur coopération aimable et patiente à cette entreprise collective.

\section{Les mécènes}

Nous remercions vivement les divers mécènes de ce livre, grâce auxquels la production et la diffusion de l'ouvrage se trouvent grandement facilitées.

\section{Muquans}

La société Muquans est une PME issue de la recherche académique, créée en 2011 et implantée dans les locaux de l'Institut d'Optique d'Aquitaine. Elle est la première au monde à proposer des solutions industrielles basées

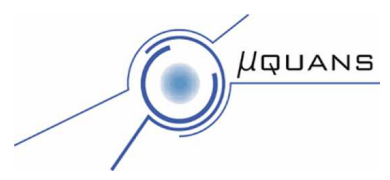
sur la manipulation quantique d'atomes froids. Elle a ainsi développé une large gamme de solutions technologiques de très haute performance et se positionne sur différents domaines d'activités : elle propose un gravimètre quantique absolu destiné au monde de la géophysique, une horloge atomique de très haute stabilité et des équipements de transfert de fréquence sur fibre sans équivalents sur le marché de la métrologie temps-fréquence, ou encore des systèmes laser intelligents parfaitement adaptés aux besoins de la physique quantique.

\section{GDR Atomes froids}

Le GdR «Atomes froids » (Groupement de Recherche du CNRS "Atomes froids») a été créé en 2012. Ce réseau de plus de 20 laboratoires à travers la France coordonne les activités dans le domaine des atomes froids à l'échelle nationale, anime la formation des jeunes doctorants, organise des rencontres et des colloques, distribue des moyens et contribue au rayonne-

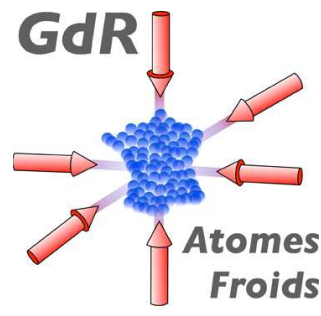
ment du domaine. 


\section{LabEx FIRST-TF}

Le LabEx FIRST-TF (« Réseau pour la Recherche, l’Innovation, la Formation, les Services et le Transfert en Temps-Fréquence ») est un réseau thématique visant à regrouper tous les acteurs du Temps-Fréquence à l'échelle nationale (20 laboratoires, 27 entreprises, 5 agences techniques et 5 autres structures). Il favorise l'émergence de projets collaboratifs, avec un spectre large d'applications, de la physique fondamentale aux systèmes de positionnement par satellite.

\section{Remerciements}

Nous remercions tout particulièrement Alain Aspect, contributeur majeur aux recherches sur les atomes froids, pour avoir accepté de rédiger une préface passionnante et très documentée qui retrace l'évolution du domaine au cours des dernières décennies.

Les remerciements des auteurs vont aux personnes suivantes : Ennio Arimondo, Hans Bachor, France Citrini, Pierre Cladé, Jean Dalibard, Dominique Delande, Antoine Heidmann, Agnès Henri, Lucile Julien, Michel Le Bellac, Lucie Marignac, Pierre Pillet, Jean-Michel Raimond, Christophe Salomon, Jook Walraven, Christoph Westbrook, Tarik Yefsah. 